\title{
BRST invariant $d=2$ condensates in Gribov-Zwanziger theory
}

\author{
Caroline Felix ${ }^{* \dagger}$ \\ KU Leuven Campus Kortrijk - Kulak, Department of Physics, Etienne Sabbelaan 53 bus 7657, \\ 8500 Kortrijk, Belgium. \\ E-mail: caroline.felix@kuleuven.be
}

\section{David Dudal}

KU Leuven Campus Kortrijk - Kulak, Department of Physics, Etienne Sabbelaan 53 bus 7657, 8500 Kortrijk, Belgium.

E-mail: david.dudalekuleuven.be

\section{Leticia Palhares}

Instituto de Física Teórica, Rua São Francisco Xavier 524, 20550-013, Maracanã, Rio de Janeiro, Brasil

E-mail: leticia.palhares@uerj.br

\section{François Rondeau}

Ecole Normale Supérieure Paris-Saclay, Avenue du Président Wilson 61, 94235 Cachan Cedex, France.

E-mail: francois.rondeaudens-cachan.fr

\section{David Vercauteren}

Duy Tân University, Institute of Research and Development, P809, 3 Quang Trung, Hải Châu, Đà Nã̃ng, Vietnam.

E-mail: vercauterendavidedtu.edu.vn

In this proceeding, $\mathrm{SU}(\mathrm{N})$ Yang-Mills theory is quantized in the linear covariant gauges, while taking into account the issue of Gribov copies, we construct the one-loop effective potential for a set of mass dimension 2 condensates, including the Gribov parameter, that refines the infrared region of the Gribov-Zwanziger theory, whilst respecting the renormalization group invariance and BRST symmetry.

XIII Quark Confinement and the Hadron Spectrum - Confinement2018

31 July - 6 August 2018

Maynooth University, Ireland

\footnotetext{
*Speaker.

${ }^{\dagger}$ Fellowship from CNPq, Brazil.
} 


\section{Introduction}

The analytical explanation of quark and gluon confinement has been a big challenge in recent decades. At low temperatures (or in the infrared regime, IR), where confinement happens, the coupling constant $\mathrm{g}^{2}$ is large, hence the perturbative formalism can not be used in this regime. This research main focus has been on the gluon propagator and also ghost propagator in the IR. The gluon propagator is suppressed to a nonvanishing value at zero momentum violating the positivity and the ghost propagator is not enhanced at at large volume according to the lattice data [1]. A possible analytical explanation for this behavior is gotten through the adding of dimension 2 condensates to the Gribov-Zwanziger (GZ) formalism, yielding to so-called Refined Gribov-Zwanziger (RGZ) framework [2,3] that fits with the lattice data quite well [1].

In this present study, the analysis of a non-trivial minimum of the effective action, which leads us to a dynamical transformation of the GZ action into the RGZ action, has been done in the presence of the $\left\langle A^{h} A^{h}\right\rangle$ and $\langle\bar{\varphi} \varphi\rangle$ condensates at one-loop following earlier steps of [4], suitably generalized to respect BRST invariance following recent developments by some of us in the field.

In this proceeding, we highlight a few steps, a comprehensive paper will be presented elsewhere.

\section{The Gribov-Zwanziger action in the linear covariant gauge}

In the IR region, the Gribov copies appear. Since the coupling constant $\mathrm{g}^{2}$ is large, these copies can not be eliminated [5]. A way to work around this problem is to restrict the functional integral to a specific region $\Omega$ in field space, a solution proposed by Gribov using the Landau gauge [5]. Moreover, this solution given by Gribov can be generalized to linear covariant gauge [6]:

$$
\Omega=\left\{A_{\mu}^{a} ; \partial_{\mu} A_{\mu}^{a}=i \alpha b^{a}, \quad \mathcal{M}^{a b}\left(A^{h}\right)=-\partial_{\mu} D_{\mu}^{a b}\left(A^{h}\right)>0\right\} .
$$

whereby the Hermitian Faddeev-Popov-related operator, $\mathcal{M}^{a b}\left(A^{h}\right)=-\delta^{a b} \partial^{2}+g f^{a b c}\left(A^{h}\right)_{\mu}^{c} \partial_{\mu}$, is positive. In (2.1), $A_{\mu}^{h}$ is a non-local power series in the gauge field, gotten from the minimization of the functional $f_{A}[u]$ along the gauge orbit of $A_{\mu}[7,8,9]$,

$$
\begin{aligned}
f_{A}[u] & \equiv \min _{\{u\}} \operatorname{Tr} \int d^{4} \chi A_{\mu}^{u} A_{\mu}^{u}, \\
A_{\mu}^{u} & =u^{\dagger} A_{\mu} u+\frac{i}{g} u^{\dagger} \partial_{\mu} u .
\end{aligned}
$$

A local minimum is found and given by

$$
\begin{aligned}
& A_{\mu}^{h}=\left(\delta_{\mu \nu}-\frac{\partial_{\mu} \partial_{v}}{\partial^{2}}\right) \phi_{v}, \quad \partial_{\mu} A_{\mu}^{h}=0 \\
& \phi_{v}=A_{v}-i g\left[\frac{1}{\partial^{2}} \partial A, A_{v}\right]+\frac{i g}{2}\left[\frac{1}{\partial^{2}} \partial A, \partial_{v} \frac{1}{\partial^{2}} \partial A\right]+O\left(A^{3}\right) .
\end{aligned}
$$


Here we highlight that $A_{\mu}^{h}$ is gauge invariant order by order [6]. The field $A_{\mu}^{h}$ can be localized by introducing an auxiliary Stueckelberg field $\xi^{\mathrm{a}}[6,10]$,

$$
A_{\mu}^{h}=\left(A^{h}\right)_{\mu}^{a} T^{a}=h^{\dagger} A_{\mu}^{a} T^{a} h+\frac{i}{g} h^{\dagger} \partial_{\mu} h,
$$

while

$$
h=e^{i g \xi^{a} T^{a}},
$$

Now, the local gauge invariance of $A_{\mu}^{h}$ under a gauge transformation $u \in S U(N)$ can be obtained from

$$
h \rightarrow u^{\dagger} h, \quad h^{\dagger} \rightarrow h^{\dagger} u, \quad A_{\mu} \rightarrow u^{\dagger} A_{\mu} u+\frac{i}{g} u^{\dagger} \partial_{\mu} u .
$$

Now, considering the BRST invariance, the Gribov-Zwanziger action in the linear covariant gauges, the total action is given by

$$
\mathrm{S}=\mathrm{S}_{\mathrm{YM}}+\mathrm{S}_{\mathrm{GF}}+\mathrm{S}_{\mathrm{GZ}}+\mathrm{S}_{\varepsilon}
$$

whereby $S_{Y M}$ is the Yang-Mills action,

$$
S_{Y M}=\frac{1}{4} \int d^{4} \chi F_{\mu \nu}^{a} F_{\mu \nu}^{a}
$$

$\mathrm{S}_{\mathrm{GF}}$ is the Faddeev-Popov gauge-fixing in linear covariant gauges,

$$
S_{G F}=\int d^{4} \chi\left(\frac{\alpha}{2} b^{a} b^{a}+i b^{a} \partial_{\mu} A_{\mu}^{a}+\bar{c}^{a} \partial_{\mu} D_{\mu}^{a b}(A) c^{b}\right)
$$

$\alpha$ being the gauge parameter and $\alpha=0$ in Landau gauge; $S_{G Z}$ is the Gribov-Zwanziger action in its local form,

$$
\begin{aligned}
S_{G Z}= & \int d^{4} x\left[\bar{\varphi}_{\mu}^{a c} \partial_{\nu} D_{\nu}^{a b}\left(A^{h}\right) \varphi_{\mu}^{b c}-\bar{\omega}_{\mu}^{a c} \partial_{\nu}\left(D_{\nu}^{a b}\left(A^{h}\right) \omega_{\mu}^{b c}\right)\right] \\
& -\gamma^{2} g \int d^{4} x\left[f^{a b c}\left(A^{h}\right)_{\mu}^{a} \varphi_{\mu}^{b c}+f^{a b c}\left(A^{h}\right)_{\mu}^{a} \bar{\varphi}_{\mu}^{b c}+\frac{d}{g}\left(N_{c}^{2}-1\right) \gamma^{2}\right]
\end{aligned}
$$

with $\left(\bar{\varphi}_{\mu}^{\mathrm{ac}}, \varphi_{\mu}^{\mathrm{ac}}\right)$ a pair of complex-conjugate bosonic fields, $\left(\bar{\omega}_{\mu}^{\mathrm{ac}}, \omega_{\mu}^{\mathrm{ac}}\right)$ a pair of anti-commuting complex-conjugate fields; and $\gamma$ the Gribov parameter which is dynamically fixed by a gap equation that gives us the horizon function [11, 12],

$$
\left\langle f^{a b c}\left(A^{h}\right)_{\mu}^{a}\left(\varphi_{\mu}^{b c}+\bar{\varphi}_{\mu}^{b c}\right)\right\rangle=2 d\left(N^{2}-1\right) \frac{\gamma^{2}}{g^{2}},
$$

which can also be rewritten as [4]

$$
\frac{\partial \Gamma}{\partial \gamma^{2}}=0
$$

whereby $\Gamma$ is the quantum action defined by

$$
\mathrm{e}^{-\Gamma}=\int[\mathrm{d} \Phi] \mathrm{e}^{-\mathrm{S}}
$$

The last term from (2.7),

$$
S_{\varepsilon}=\int d^{4} x \varepsilon^{a} \partial_{\mu}\left(A^{h}\right)_{\mu}^{a}
$$


ensures, through the Lagrange multiplier $\varepsilon$, the transversality of the composite operator $\left(A^{h}\right)_{\mu}^{a}$, $\partial_{\mu}\left(A^{h}\right)_{\mu}^{a}=0$.

The action $S$, (2.7), enjoys an exact BRST invariance, $s S=0$ and $s^{2}=0$ [6]

$$
\begin{array}{ll}
s A_{\mu}^{a}=-D_{\mu}^{a b} c^{b}, & s c^{a}=\frac{g}{2} f^{a b c} c^{b} c^{c}, \\
s \bar{c}^{a}=i b^{a}, & s b^{a}=0, \\
s \varphi_{\mu}^{a b}=0, & s \omega_{\mu}^{a b}=0, \\
s \bar{\omega}_{\mu}^{a b}=0, & s \bar{\varphi}_{\mu}^{a b}=0, \\
s \varepsilon^{a}=0, & s\left(A^{h}\right)_{\mu}^{a}=0, \\
s h^{i j}=-i g c^{a}\left(T^{a}\right)^{i k} h^{k j} .
\end{array}
$$

\section{Refined Gribov-Zwanziger Action}

The BRST invariant $d=2$ condensates, $\left\langle A_{h, \mu}^{a} A_{h, \mu}^{a}\right\rangle$ and $\left\langle\bar{\varphi}_{\mu}^{a b} \varphi_{\mu}^{a b}\right\rangle$, cause non-perturbative dynamical instabilities disturbing the Gribov-Zwanziger formalism $[2,3,4] .\left\langle\bar{\varphi}_{\mu}^{a b} \varphi_{\mu}^{a b}\right\rangle$ guarantees that the gluon propagator is non-vanishing at zero momentum, and $\left\langle A_{\mu}^{a} A_{\mu}^{a}\right\rangle$ assures to fit the result with the lattice data [4]. The refined Gribov-Zwanziger action (RGZ) is obtained adding these condensates to the GZ action via the local composite operator (LCO) formalism, see [4]. The operators $A^{\mathrm{h}} A^{\mathrm{h}}$ and $\bar{\varphi} \varphi$ will be added to the action via two BRST invariant bosonic sources $\tau$ and Q,

$$
s \tau=0 \quad \text { and } \quad s Q=0 .
$$

From here, we opted for the Landau gauge $\partial A=0$ for convenience, so that we can work with $A^{h}=A$, as formally proven in [6]. Then, the action with these operators is written as

$$
\Sigma=\mathrm{S}+\mathrm{S}_{\mathrm{A}^{2}}+\mathrm{S}_{\varphi \bar{\varphi}}+\mathrm{S}_{\mathrm{vac}}
$$

whereby $S$ is given by (2.7) and we also have

$$
\begin{aligned}
S_{A^{2}} & =\int d^{\mathcal{D}} \chi \frac{\tau}{2} A_{\mu}^{a} A_{\mu}^{a}, \\
S_{\bar{\varphi} \varphi} & =\int d^{\mathcal{D}} \chi Q \bar{\varphi} \bar{\varphi}_{\mu}^{a c} \varphi_{\mu}^{a c}, \\
S_{\text {vac }} & =-\int d^{\mathcal{D}} \chi\left(\frac{\zeta}{2} \tau^{2}+\alpha Q Q+\chi Q \tau\right) .
\end{aligned}
$$

The parameters $\alpha, \chi$ and $\zeta$ are the LCO parameters which guarantee that the divergences of the kind $\left\langle A^{2}(x) A^{2}(y)\right\rangle_{x \rightarrow y}$, etc. can be properly dealt with, see [4].

\section{The Effective Action Calculus}

In order to get the effective action, we have written the energy functional as

$$
e^{-W(J)}=\int[d \Phi] e^{-\Sigma}
$$


where $\Sigma$ is given by (3.2).

The action $\Sigma$, (3.2), has three terms quadratic in the sources and they should be removed to facilitate calculations and interpret our results in terms of the vacuum energy. The easiest way to remove these terms is by introducing two auxiliary fields $\sigma_{1}$ and $\sigma_{2}$ via two identities

$$
\begin{aligned}
& 1=\int\left[\mathcal{D} \sigma_{1}\right] e^{-\frac{1}{2} \int d^{d} x\left(\sigma_{1}+\frac{\bar{a}}{2} A^{2}+\bar{b} Q+\bar{c} \tau\right)^{2}}, \\
& 1=\int\left[D \sigma_{2}\right] e^{+\frac{1}{2} \int d^{d} x\left(\sigma_{2}+\bar{d} \bar{\varphi} \varphi+\bar{e} Q+\frac{\bar{f}}{2} A^{2}\right)^{2}},
\end{aligned}
$$

multiplying the integral in (4.1). If we choose

$$
\begin{aligned}
& \overline{\mathrm{a}}=-\frac{Z_{\mathrm{A}}}{\sqrt{Z_{\zeta \zeta \zeta}}} \mu^{\epsilon / 2} \\
& \overline{\mathrm{b}}=\frac{\mathrm{Z}_{\mathrm{QQ}} Z_{\chi \chi} \chi}{\sqrt{Z_{\zeta \zeta \zeta}}} \mu^{-\epsilon / 2}, \\
& \bar{c}=Z_{\tau \tau} \sqrt{Z_{\zeta \zeta \zeta}} \mu^{-\epsilon / 2}, \\
& \overline{\mathrm{d}}=\frac{Z_{\varphi}}{\sqrt{\frac{Z_{\chi \chi}^{2} \chi^{2}}{Z_{\zeta \zeta \zeta}}-2 Z_{\alpha \alpha} \alpha}} \mu^{\epsilon / 2}, \\
& \bar{e}=Z_{Q Q} \sqrt{\frac{Z_{\chi \chi}^{2} \chi^{2}}{Z_{\zeta \zeta \zeta}}-2 Z_{\alpha \alpha} \alpha \mu^{-\epsilon / 2}}, \\
& \bar{f}=\frac{Z_{\mathrm{A}}}{\sqrt{Z_{\zeta \zeta \zeta}}}\left(\frac{Z_{\tau Q} Z_{\zeta \zeta \zeta-} Z_{Q Q} Z_{\chi \chi} \chi}{Z_{Q Q} \sqrt{Z_{\chi \chi}^{2} \chi^{2}-2 Z_{\alpha \alpha} Z_{\zeta \zeta \zeta \alpha}}}\right) \mu^{\epsilon / 2},
\end{aligned}
$$

we can remove the quadratic terms in sources. In the $\overline{\mathrm{MS}}$ scheme and at one loop, the $\mathrm{Z}$ factors are given by [4]

$$
\begin{aligned}
Z_{A} & =1+\frac{13}{3} \frac{\mathrm{Ng}^{2}}{16 \pi^{2} \epsilon}, & \tilde{Z}_{\zeta}=Z_{\zeta \zeta} Z_{\tau \tau}^{2}=1-\frac{13}{3} \frac{\mathrm{Ng}^{2}}{16 \pi^{2} \epsilon}, & Z_{\zeta \zeta}=1+\frac{22}{3} \frac{\mathrm{Ng}^{2}}{16 \pi^{2} \epsilon} \\
Z_{g} & =1-\frac{11}{3} \frac{\mathrm{Ng}^{2}}{16 \pi^{2} \epsilon}, & Z_{\tau \tau}=1-\frac{35}{6} \frac{\mathrm{Ng}^{2}}{16 \pi^{2} \epsilon}, \quad Z_{Q Q}=Z_{g} Z_{A}^{1 / 2}=1-\frac{3}{2} \frac{\mathrm{Ng}^{2}}{16 \pi^{2} \epsilon}, & Z_{\alpha}=Z_{\alpha \alpha} Z_{Q Q}^{2}=1+\frac{35}{6} \frac{\mathrm{Ng}^{2}}{16 \pi^{2} \epsilon}, \\
Z_{\chi \chi} & =1, & Z_{\tau Q}=0, & Z_{\varphi}=Z_{\bar{\varphi}}=Z_{g}^{-1} Z_{A}^{-1 / 2}=1+\frac{3}{2} \frac{\mathrm{Ng}^{2}}{16 \pi^{2} \epsilon} .
\end{aligned}
$$

Therefore, (4.1) becomes

$$
\begin{aligned}
& e^{-W(Q, \tau)}=\int[\mathcal{D} \Phi]\left[\mathcal{D} \sigma_{1,3}\right] \exp \left[-S_{G Z}-\frac{1}{2} \int d^{d} x\left(2 \bar{c} \sigma_{1} \tau+2 \sigma_{3} Q\left(1-\frac{\bar{b}^{2}}{\bar{e}^{2}}\right) \sigma_{1}^{2}\right.\right. \\
& -\frac{1}{\bar{e}^{2}}\left(\sigma_{3}^{2}-2 \bar{b} \sigma_{1} \sigma_{3}\right)+\left(\left(\bar{a}-\frac{\bar{f} \bar{b}}{\bar{e}}\right)\left\langle\sigma_{1}\right\rangle+\frac{\bar{f}}{\bar{e}}\left\langle\sigma_{3}\right\rangle\right) A^{2} \\
& \left.\left.-2 \frac{\overline{\mathrm{d}}}{\overline{\mathrm{e}}}\left(\overline{\mathrm{b}}\left\langle\sigma_{1}\right\rangle-\left\langle\sigma_{3}\right\rangle\right) \bar{\varphi} \varphi\right)\right]
\end{aligned}
$$


where

$$
\sigma_{3}=\sigma_{1} \bar{b}-\sigma_{2} \bar{e} .
$$

So far, all LCO parameters, sources and fields have been renormalized, except the auxiliary fields

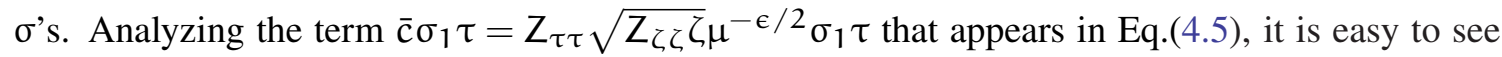
that this field is indeed infinite, thus it also must be renormalized. As the Z-factors are infinite, if $\sigma_{1}$ would be finite, then the quantity multiplying $\tau$ would be infinite. This would not make sense as a physical (and thus finite) local operator $\mathcal{O}$. The original $\sigma_{1}$ field should then be infinite in order to get a finite quantity multiplying the finite source $\tau$. It is then natural to define a renormalized finite field $\sigma_{1}^{\prime}$ by $\sigma_{1}^{\prime} \equiv Z_{\tau \tau} \sqrt{Z_{\zeta \zeta}} \sigma_{1} \equiv \sqrt{\widetilde{Z}_{\zeta}} \sigma_{1}$. Regarding $\sigma_{3}$, the term $\sigma_{3} Q$ in Eq.(4.5) teach us, knowing that $\mathrm{Q}$ is finite, that $\sigma_{3}$ is already finite and thus should not be renormalized. In terms of the finite fields $\sigma_{1}^{\prime}$ and $\sigma_{3}$, the energy functional now reads :

$$
\begin{aligned}
& e^{-W(Q, \tau)} S=\int[\mathcal{D} \Phi]\left[\mathcal{D} \sigma_{1,3}\right] \exp \left[-S_{G Z}-\frac{1}{2} \int d^{d} x\left(-2 \sqrt{\zeta} \sigma_{1}^{\prime} \tau+2 \sigma_{3} \mathrm{Q}\left(1-\frac{\bar{b}^{2}}{\bar{e}^{2}}\right) \frac{\sigma_{1}^{\prime 2}}{\widetilde{Z}_{\zeta}}\right.\right. \\
& -\frac{1}{\bar{e}^{2}}\left(\sigma_{3}^{2}-2 \bar{b} \frac{\sigma_{1}^{\prime}}{\sqrt{\widetilde{Z}_{\zeta}}} \sigma_{3}\left(\bar{a}-\frac{\bar{f} \bar{b}}{\bar{e}}\right) \frac{\left\langle\sigma_{1}^{\prime}\right\rangle}{\sqrt{\widetilde{Z}_{\zeta}}}+\frac{\bar{f}}{\bar{e}}\left\langle\sigma_{3}\right\rangle\right) A^{2}
\end{aligned}
$$

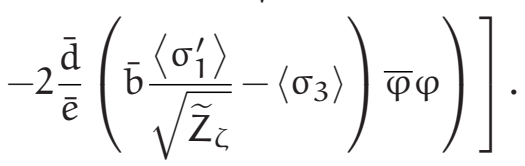

In this expression, all LCO parameters, sources and fields are now finite, and infinities are only present in the renormalization factors $Z$ 's, explicitly written or present in the coefficients $\overline{\mathrm{a}}, \ldots, \overline{\mathrm{f}}$. At one loop, $\chi=0, Z_{\tau Q}=0$ [4] which implies that $\bar{b}=\bar{f}=0$, then $\sigma_{3}=-\bar{e} \sigma_{2}$. Now, by analysis of the term $\sigma_{3} \mathrm{Q}=-\overline{\mathrm{e}} \sigma_{2} \mathrm{Q}$ in (4.7), a same reasoning as above for $\sigma_{1}$ shows that $\sigma_{2}$ is infinite and should be renormalized defining a new finite field $\sigma_{2}^{\prime}$ through $\sigma_{2}^{\prime} \equiv Z_{Q Q} \sqrt{Z_{\alpha \alpha}} \sigma_{2} \equiv \sqrt{\widetilde{Z_{\alpha}}} \sigma_{2}$. Hence, the energy functional in terms of the finite fields $\sigma_{1}^{\prime}$ and $\sigma_{2}^{\prime}$ and with one-loop coefficients reads :

$$
\begin{aligned}
e^{-W(Q, \tau)}=\int[\mathcal{D} \Phi]\left[\mathcal{D} \sigma_{1,2}\right] \exp & {\left[-S_{\mathrm{GZ}}-\frac{1}{2} \int \mathrm{d}^{\mathrm{d}} \chi\left(\frac{\sigma_{1}^{\prime 2}}{\widetilde{\mathrm{Z}}_{\zeta}}-\frac{\sigma_{2}^{\prime 2}}{\widetilde{\mathrm{Z}}_{\alpha}}+\overline{\mathrm{a}} \frac{\left\langle\sigma_{1}^{\prime}\right\rangle}{\sqrt{\widetilde{Z}_{\zeta}}} \mathrm{A}^{2}-2 \overline{\mathrm{d}} \frac{\left\langle\sigma_{2}^{\prime}\right\rangle}{\sqrt{\widetilde{Z}_{\alpha}}} \bar{\varphi} \varphi\right.\right.} \\
& \left.\left.-2 \sqrt{\zeta} \sigma_{1}^{\prime} \tau+2 \sqrt{-2 \alpha} \sigma_{2}^{\prime} \mathrm{Q}\right)\right]
\end{aligned}
$$

In this expression, infinities are now localized in four different places, only in the renormalization factors $\tilde{Z}_{\zeta}, \tilde{Z}_{\alpha}$, and in those hidden in $\bar{a}$ and $\bar{d}$.

In order to have an expression of the form $\frac{m^{2}}{2} A^{2}-M^{2} \bar{\varphi} \varphi$, we defined the effective masses, $\mathrm{m}^{2}$ and $\mathrm{M}^{2}$, respectively linked to $\langle A A\rangle$ and $\langle\bar{\varphi} \varphi\rangle$ by:

$$
\mathrm{m}^{2} \equiv \frac{\overline{\mathrm{a}}}{\sqrt{\widetilde{\mathrm{Z}_{\zeta}}}}\left\langle\sigma_{1}^{\prime}\right\rangle=\left(1+\frac{17}{6} \frac{\mathrm{Ng}^{2}}{16 \pi^{2} \epsilon}\right) \sqrt{\frac{13 \mathrm{Ng}^{2}}{9\left(\mathrm{~N}^{2}-1\right)}}\left\langle\sigma_{1}^{\prime}\right\rangle+\mathcal{O}\left(\mathrm{g}^{4}\right)
$$




$$
M^{2} \equiv \frac{\overline{\mathrm{d}}}{\sqrt{\widetilde{\mathrm{Z}_{\alpha}}}}\left\langle\sigma_{2}^{\prime}\right\rangle=-\left(1-\frac{35}{6} \frac{\mathrm{Ng}^{2}}{16 \pi^{2} \epsilon}\right) \sqrt{\frac{35 \mathrm{Ng}^{2}}{48\left(\mathrm{~N}^{2}-1\right)^{2}}}\left\langle\sigma_{2}^{\prime}\right\rangle+\mathcal{O}\left(\mathrm{g}^{4}\right)
$$

where the last equalities follow from considering the first order term of the Z-factors in $\overline{\mathrm{MS}}$ scheme and $\alpha=\frac{\alpha_{0}}{g^{2}}=-\frac{24\left(\mathrm{~N}^{2}-1\right)^{2}}{35 \mathrm{Ng}^{2}}$ and $\zeta=\frac{\zeta_{0}}{g^{2}}=\frac{9\left(\mathrm{~N}^{2}-1\right)}{13 \mathrm{Ng}^{2}}[4]$.

The ghost fields $c, \bar{c}, \omega, \bar{\omega}$ give us just an overall factor. Now, to integrate over the $\varphi$ and $\bar{\varphi}$ fields, we proceed as follows

$$
\bar{\varphi}_{\mu}^{a b}=U_{\mu}^{a b}+i V_{\mu}^{a b}, \varphi_{\mu}^{a b}=U_{\mu}^{a b}-i V_{\mu}^{a b} .
$$

Then

$$
\begin{aligned}
& \int[\mathcal{D U}, \mathrm{V}] \quad e^{-\int \mathrm{d}^{\mathrm{d}} x\left[\mathrm{~V}_{\mu}^{\mathrm{ab}} P_{\mu \nu}^{a c, b d} V_{\nu}^{c d}+\mathrm{U}_{\mu}^{\mathrm{ab}} P_{\mu \nu}^{a c, b d} U_{\nu}^{c d}-2 g \gamma^{2} f^{a b c} A_{\mu}^{a} U_{\mu}^{b c}\right]}= \\
& =\frac{1}{\operatorname{det} P_{\mu \nu}^{a c}, b d} e^{\int d^{d} \times N g^{2} \gamma^{4} A_{\mu}^{a} P_{\mu \nu}^{-1} \delta^{a b} A_{\nu}^{b}},
\end{aligned}
$$

with

$$
P_{\mu \nu}^{a c, b d} \equiv\left(\partial^{2}-M^{2}\right) \delta^{a c} \delta^{b d} \delta_{\mu \nu} .
$$

Therefore, the first contribution $\Gamma_{a}$ to the effective potential is obtained by :

$$
\Omega \Gamma_{\mathrm{a}}=\ln \operatorname{det} \mathrm{P}_{\mu \nu}^{\mathrm{ac}, \mathrm{bd}}=\operatorname{Tr} \ln \mathrm{P}_{\mu \nu}^{\mathrm{ac}, \mathrm{bd}},
$$

resulting in

$$
\begin{aligned}
\Gamma_{\mathrm{a}} & =\left(\mathrm{N}^{2}-1\right)^{2}\left[-\frac{1}{\epsilon} \frac{\mathrm{M}^{4}}{4 \pi^{2}}+\frac{\mathrm{M}^{4}}{8 \pi^{2}} \ln \frac{\mathrm{M}^{2}}{\bar{\mu}^{2}}-\frac{\mathrm{M}^{4}}{8 \pi^{2}}\right] \\
& =\frac{35 \mathrm{Ng}^{2}}{48} \frac{\left\langle\sigma_{2}^{\prime}\right\rangle^{2}}{4 \pi^{2}}\left(-\frac{1}{\epsilon}-\frac{1}{2}+\frac{1}{2} \ln \left(-\sqrt{\frac{35 \mathrm{Ng}^{2}}{48\left(\mathrm{~N}^{2}-1\right)^{2}}} \frac{\left\langle\sigma_{2}^{\prime}\right\rangle}{\bar{\mu}^{2}}\right)\right)+\mathcal{O}\left(\mathrm{g}^{4}\right),
\end{aligned}
$$

whereby $\bar{\mu}$ is the energy scale. The second contribution $\Gamma_{\mathrm{b}}$ to the effective potential comes from the gluon field $A_{\mu}$. The quadratic part of the action containing $A_{\mu}$ is

$$
e^{-\frac{1}{2} \int d^{d} \chi A_{\mu}^{a} R_{\mu \nu}^{a b} A_{\nu}^{b}}
$$

where

$$
R_{\mu \nu}^{a b} \equiv \delta^{a b}\left[\left(-\partial^{2}+m^{2}-\frac{2 N \gamma^{4} g^{2}}{\partial^{2}-M^{2}}\right) \delta_{\mu \nu}-\partial_{\mu} \partial_{\nu}\left(\frac{1}{\alpha}-1\right)\right] .
$$

Therefore,

$$
\Omega \Gamma_{\mathrm{b}}=\frac{1}{2} \ln \operatorname{det} R_{\mu \nu}^{\mathrm{ac}, \mathrm{bd}}=\frac{1}{2} \operatorname{Tr} \ln R_{\mu \nu}^{\mathrm{ac}, \mathrm{bd}}
$$

resulting in

$$
\begin{aligned}
\Gamma_{\mathrm{b}}= & -\frac{\left(\mathrm{N}^{2}-1\right)}{2(4 \pi)^{2}}\left(\frac{3}{\epsilon}+\frac{5}{4}\right)\left(\mathrm{m}^{4}-4 \gamma^{4} g^{2} \mathrm{~N}\right)+\frac{3\left(\mathrm{~N}^{2}-1\right)}{4(4 \pi)^{2}}\left[x_{1}^{2} \ln \left(\frac{-\chi_{1}}{\bar{\mu}^{2}}\right)+x_{2}^{2} \ln \left(\frac{-\chi_{2}}{\bar{\mu}^{2}}\right)\right. \\
& \left.-M^{4} \ln \left(\frac{M^{2}}{\bar{\mu}^{2}}\right)\right],
\end{aligned}
$$


where $x_{1}$ and $x_{2}$ are the solutions of the equation $x^{2}+\left(M^{2}+m^{2}\right) x+M^{2} m^{2}+\lambda^{4}=0$,

$$
\begin{aligned}
& x_{1}=-\frac{1}{2}\left(m^{2}+M^{2}+\sqrt{\left(m^{2}-M^{2}\right)^{2}-4 \lambda^{4}}\right), \\
& x_{2}=-\frac{1}{2}\left(m^{2}+M^{2}-\sqrt{\left(m^{2}-M^{2}\right)^{2}+-4 \lambda^{4}}\right) .
\end{aligned}
$$

The third part of effective potential $\Gamma_{\mathrm{c}}$ is from the Gribov-Zwanziger action,

$$
\Gamma_{\mathrm{c}}=-\mathrm{d} \gamma_{0}^{4}\left(\mathrm{~N}^{2}-1\right) \text {. }
$$

Knowing that $Z_{\gamma^{2}}=Z_{g}^{-1 / 2} Z_{A}^{-1 / 4}$, we get

$$
\gamma_{0}^{4}=Z_{\gamma^{2}}^{2} \gamma^{4}, \text { with } Z_{\gamma^{2}}^{2}=1+\frac{3}{2} \frac{g^{2} N}{16 \pi^{2} \epsilon}
$$

hence

$$
\Gamma_{\mathrm{c}}=\left(\mathrm{N}^{2}-1\right) \gamma^{4}\left(-4+\frac{3 \mathrm{Ng}}{32 \pi^{2}}-\frac{3 \mathrm{Ng}^{2}}{8 \pi^{2} \epsilon}\right)+\mathcal{O}\left(\mathrm{g}^{4}\right) .
$$

And the last contribution comes from $\left\langle\sigma_{1}^{\prime}\right\rangle^{2}$ and $\left\langle\sigma_{2}^{\prime}\right\rangle^{2}$ :

$$
\begin{aligned}
\Gamma_{\mathrm{d}} & =\frac{1}{2}\left(\frac{1}{\tilde{Z}_{\zeta}}\left\langle\sigma_{1}^{\prime}\right\rangle^{2}-\frac{1}{\tilde{Z}_{\alpha}}\left\langle\sigma_{2}^{\prime}\right\rangle^{2}\right) \\
& =\frac{\left\langle\sigma_{1}^{\prime}\right\rangle^{2}}{2}-\frac{\left\langle\sigma_{2}^{\prime}\right\rangle^{2}}{2}+\frac{13}{6} \frac{\mathrm{Ng}^{2}}{16 \pi^{2} \epsilon}\left\langle\sigma_{1}^{\prime}\right\rangle^{2}+\frac{35}{12} \frac{\mathrm{Ng}^{2}}{16 \pi^{2} \epsilon}\left\langle\sigma_{2}^{\prime}\right\rangle^{2}+\mathcal{O}\left(\mathrm{g}^{4}\right) .
\end{aligned}
$$

The full effective potential given by $\Gamma=\Gamma_{\mathrm{a}}+\Gamma_{\mathrm{b}}+\Gamma_{\mathrm{c}}+\Gamma_{\mathrm{d}}$ is finite when $\epsilon \rightarrow 0$ at first order in $\mathrm{g}^{2}$. Therefore, it can be written as

$$
\begin{gathered}
\Gamma\left(\mathrm{m}^{2}, \mathrm{M}^{2}, \lambda^{4}\right)=\frac{9\left(\mathrm{~N}^{2}-1\right)}{13 \mathrm{Ng}^{2}} \frac{\mathrm{m}^{4}}{2}-\frac{48\left(\mathrm{~N}^{2}-1\right)^{2}}{35 \mathrm{Ng}^{2}} \frac{\mathrm{M}^{4}}{2}-\frac{2 \lambda^{4}\left(\mathrm{~N}^{2}-1\right)}{\mathrm{Ng}^{2}} \\
-\frac{\mathrm{N}^{2}-1}{16 \pi^{2}}\left\{-2 \lambda^{4}+\frac{5}{8} \mathrm{~m}^{4}+2\left(\mathrm{~N}^{2}-1\right) \mathrm{M}^{4}-\left(2\left(\mathrm{~N}^{2}-1\right)-\frac{3}{4}\right) \ln \left(\frac{\mathrm{M}^{2}}{\bar{\mu}^{2}}\right) \mathrm{M}^{4}\right\} \\
+\frac{3}{8} \frac{\mathrm{N}^{2}-1}{16 \pi^{2}}\left\{\mathrm{~m}^{4}+\mathrm{M}^{4}-2 \lambda^{4}+\left(\mathrm{m}^{2}+\mathrm{M}^{2}\right) \sqrt{\left(\mathrm{m}^{2}-\mathrm{M}^{2}\right)^{2}-4 \lambda^{4}}\right\} \\
\times \ln \left[\frac{1}{2 \bar{\mu}^{2}}\left(\mathrm{~m}^{2}+\mathrm{M}^{2}+\sqrt{\left(\mathrm{m}^{2}-\mathrm{M}^{2}\right)^{2}-4 \lambda^{4}}\right)\right] \\
+\frac{3}{8} \frac{\mathrm{N}^{2}-1}{16 \pi^{2}}\left\{\mathrm{~m}^{4}+M^{4}-2 \lambda^{4}-\left(\mathrm{m}^{2}+M^{2}\right) \sqrt{\left(\mathrm{m}^{2}-\mathrm{M}^{2}\right)^{2}-4 \lambda^{4}}\right\} \\
\times \ln \left[\frac{1}{2 \bar{\mu}^{2}}\left(\mathrm{~m}^{2}+M^{2}-\sqrt{\left(\mathrm{m}^{2}-M^{2}\right)^{2}-4 \lambda^{4}}\right)\right] .
\end{gathered}
$$

with

$$
\begin{aligned}
& m^{2}=\sqrt{\frac{13 \mathrm{Ng}^{2}}{9\left(\mathrm{~N}^{2}-1\right)}}\left\langle\sigma_{1}^{\prime}\right\rangle, \\
& M^{2}=-\sqrt{\frac{35 \mathrm{Ng}^{2}}{48\left(\mathrm{~N}^{2}-1\right)^{2}}}\left\langle\sigma_{2}^{\prime}\right\rangle .
\end{aligned}
$$


and $\lambda^{4} \equiv 2 \mathrm{Ng}^{2} \gamma^{4}$. The next step is to analyze the gap equations given by

$$
\frac{\partial \Gamma}{\partial M^{2}}=0, \quad \frac{\partial \Gamma}{\partial m^{2}}=0, \quad \frac{\partial \Gamma}{\partial \lambda^{4}}=0 .
$$

Unfortunately, an acceptable result was not obtained in this particular scheme. The resolution was to not fix a scheme, we rather rewrote the effective potential in a general scheme as has been done in e.g. [13]. Details of this procedure will be published elsewhere. The effective potential (4.25), in general scheme, becomes

$$
\begin{gathered}
\Gamma_{\text {gen }}\left(m^{2}, M^{2}, \lambda^{4}, b_{0}\right)=\frac{9\left(N^{2}-1\right)}{26 N g^{2}} m^{4}-\frac{24\left(N^{2}-1\right)^{2}}{35 N g^{2}} M^{4}-\frac{2\left(N^{2}-1\right)^{2} M^{4}}{16 \pi^{2}}\left(1-\ln \left(\frac{M^{2}}{\bar{\mu}^{2}}\right)\right) \\
-2 \lambda^{4} \frac{N^{2}-1}{N^{2}}-2 \lambda^{4} \frac{N^{2}-1}{16 \pi^{2}}\left(b_{0}-1\right) \\
+\frac{3}{4} \frac{N^{2}-1}{16 \pi^{2}}\left\{\frac{5}{4}\left(m^{4}+M^{4}-2 \lambda^{4}\right)-\frac{m^{2}+M^{-} 2 \lambda^{4}}{2} \ln \left[\frac{m^{2} M^{2}+\lambda^{4}}{\bar{\mu}^{4}}\right]\right. \\
\left.+\left(m^{2}+M^{2}\right) \sqrt{4 \lambda^{4}-\left(m^{2}-M^{2}\right)^{2}} \arctan \left[\frac{\sqrt{4 \lambda^{4}-\left(m^{2}-M^{2}\right)^{2}}}{m^{2}+M^{2}}\right]+\ln \left[\frac{M^{2}}{\bar{\mu}^{2}}\right] M^{4}\right\},
\end{gathered}
$$

$b_{0}$ being a parameter related to the chosen scheme for the coupling. It was fixed, at the end, by the matching our values for the complex conjugate poles masses of the transverse gluon propagator to those estimated from lattice data [1] when the gap equations are solved for. The effective masses $\mathrm{m}^{2}$ and $M^{2}$ and the Gribov parameter $\gamma^{2}$ were gotten in function of the parameter $b_{0}$ and $\bar{\mu}$ in units $\Lambda=1, N=3$ and also with $N=2$. Notice that these poles masses are gauge and scheme independent [6], so we benefitted from this to fix the parameters $b_{0}$ and $\bar{\mu}$ by using a minimal external lattice input to determine the "optimum scheme". We got $b_{0}=-3.643$ and $\bar{\mu}=1.429$. With this procedure, we obtained a reasonable value for the coupling constant, namely 0.382 . Therefore, in this case, the perturbative result is relatively trustworthy. The Gribov parameter $\gamma^{2}$ is 0.637 and the vacuum energy is -26.955 . The Hessian determinant is positive and also the second derivatives,

$$
\left.\frac{\partial^{2} \Gamma_{\text {gen }}}{\partial M^{2}}\right|_{\text {solved }}=\left.1.668 \quad \frac{\partial^{2} \Gamma_{\text {gen }}}{\partial m^{2}}\right|_{\text {solved }}=\left.0.216 \quad \frac{\partial^{2} \Gamma_{\text {gen }}}{\partial M^{2} \partial m^{2}}\right|_{\text {solved }}=0.011
$$

Then, the solution does correspond to a minimum.

The future step will be to extend this research to finite temperatures and to study if the deconfinement transitions reflects itself in a change in the propagator behavior and to check if, with the Polyakov loop added to the game, we observe the transition also in that order parameter.

\section{References}

[1] D. Dudal, O. Oliveira and P. J. Silva, Annals Phys. 397 (2018) 351; A. Cucchieri, D. Dudal, T. Mendes, O. Oliveira, M. Roelfs and P. J. Silva, arXiv:1809.08224 [hep-lat]; A. Cucchieri, T. Mendes and E. M. S. Santos, Phys. Rev. Lett. 103 (2009) 141602; P. Bicudo, D. Binosi, N. Cardoso, O. Oliveira and P. J. Silva, Phys. Rev. D 92 (2015) no.11, 114514; A. Cucchieri, D. Dudal, T. Mendes and N. Vandersickel, Phys. Rev. D 85 (2012) 094513. 
[2] D. Dudal, S. P. Sorella, N. Vandersickel and H. Verschelde, Phys. Rev. D 77 (2008) 071501.

[3] D. Dudal, J. A. Gracey, S. P. Sorella, N. Vandersickel and H. Verschelde, Phys. Rev. D 78 (2008) 065047 .

[4] D. Dudal, S. P. Sorella and N. Vandersickel, Phys. Rev. D 84 (2011) 065039.

[5] V. N. Gribov, Nucl. Phys. B 139 (1978) 1.

[6] M. A. L. Capri et al., Phys. Rev. D 92 (2015) 045039; M. A. L. Capri et al., Phys. Rev. D 93 (2016) 065019; M. A. L. Capri et al., Phys. Rev. D 94 (2016) 025035; M. A. L. Capri, D. Fiorentini,

A. D. Pereira, R. F. Sobreiro, S. P. Sorella and R. C. Terin, Annals Phys. 376 (2017) 40;

M. A. L. Capri, D. Dudal, A. D. Pereira, D. Fiorentini, M. S. Guimaraes, B. W. Mintz, L. F. Palhares and S. P. Sorella, Phys. Rev. D 95 (2017) 045011.

[7] G. Dell'Antonio and D. Zwanziger, Nucl. Phys. B 326 (1989) 333.

[8] P. van Baal, Nucl. Phys. B 369 (1992) 259.

[9] M. Lavelle and D. McMullan, Phys. Rept. 279 (1997) 1.

[10] M. A. L. Capri, D. Fiorentini, M. S. Guimaraes, B. W. Mintz, L. F. Palhares and S. P. Sorella, Phys. Rev. D 94 (2016) 065009.

[11] D. Zwanziger, Nucl. Phys. B 323 (1989) 513.

[12] D. Zwanziger, Nucl. Phys. B 399 (1993) 477.

[13] D. Dudal, R. F. Sobreiro, S. P. Sorella and H. Verschelde, Phys. Rev. D 72 (2005) 014016. 